\title{
Experimental Demonstration of multiple Disaggregated OLTs with Virtualised Multi Tenant DBA, over General Purpose Processor
}

\author{
Frank Slyne $^{(1)}$, David Coyle ${ }^{(2)}$, Jasvinder Singh ${ }^{(2)}$, Rory Sexton ${ }^{(2)}$, Brendan Ryan ${ }^{(2)}$, \\ Robin Giller $^{(2)}$, Michael O'Hanlon ${ }^{(2)}$, Marco Ruffini ${ }^{(1)}$ \\ ${ }^{(1)}$ CONNECT Centre, Trinity College Dublin, Ireland, ${ }^{(2)}$ Intel Corporation, Ireland \\ $\{$ slyne,marco.ruffini $\} @$ tcd.ie, \{david.coyle, jasvinder.singh, rory.sexton,brendan.ryan, \\ robin.giller,michael.a.ohanlon\}@intel.com
}

\begin{abstract}
We demonstrate an Optical Line Terminal with fully softwarised data plane and virtual Dynamic Bandwidth Allocation in a sliceable, multi-tenant PON architecture. We evaluate performance results for 6 OLTs sharing the same general purpose processor. (C) 2020 The Author(s)
\end{abstract}

\section{Overview}

The economic viability of future 5th Generation networks depends on the availability of ubiquitous high speed networks. Passive Optical Networks (PONs) can play a key role as the bearer of multiple heterogeneous services for high speed networks, particularly when sliced across multiple virtual operators running diverse services.

There are currently a number of active projects, such as OpenOLT [1] whose aim is to increase sharing of physical Optical Line Terminal (OLT) infrastructure. In our demonstration, we deliver a performant OLT implementation entirely in software that enables innovation through virtualisation of the DBA function. We demonstrate the technical feasibility of running 6 XGS-PON OLT dataplanes on a single Xeon General Purpose Processor. Upstream bandwidth allocation for each of the 6 PONs is administered by a virtualised Dynamic Bandwidth Allocation (DBA) kernel running on the same Xeon server. The performance of the virtualised OLT and DBA is greatly improved through the use of the Data Plane Development Kit (DPDK) dataplane acceleration toolkit.

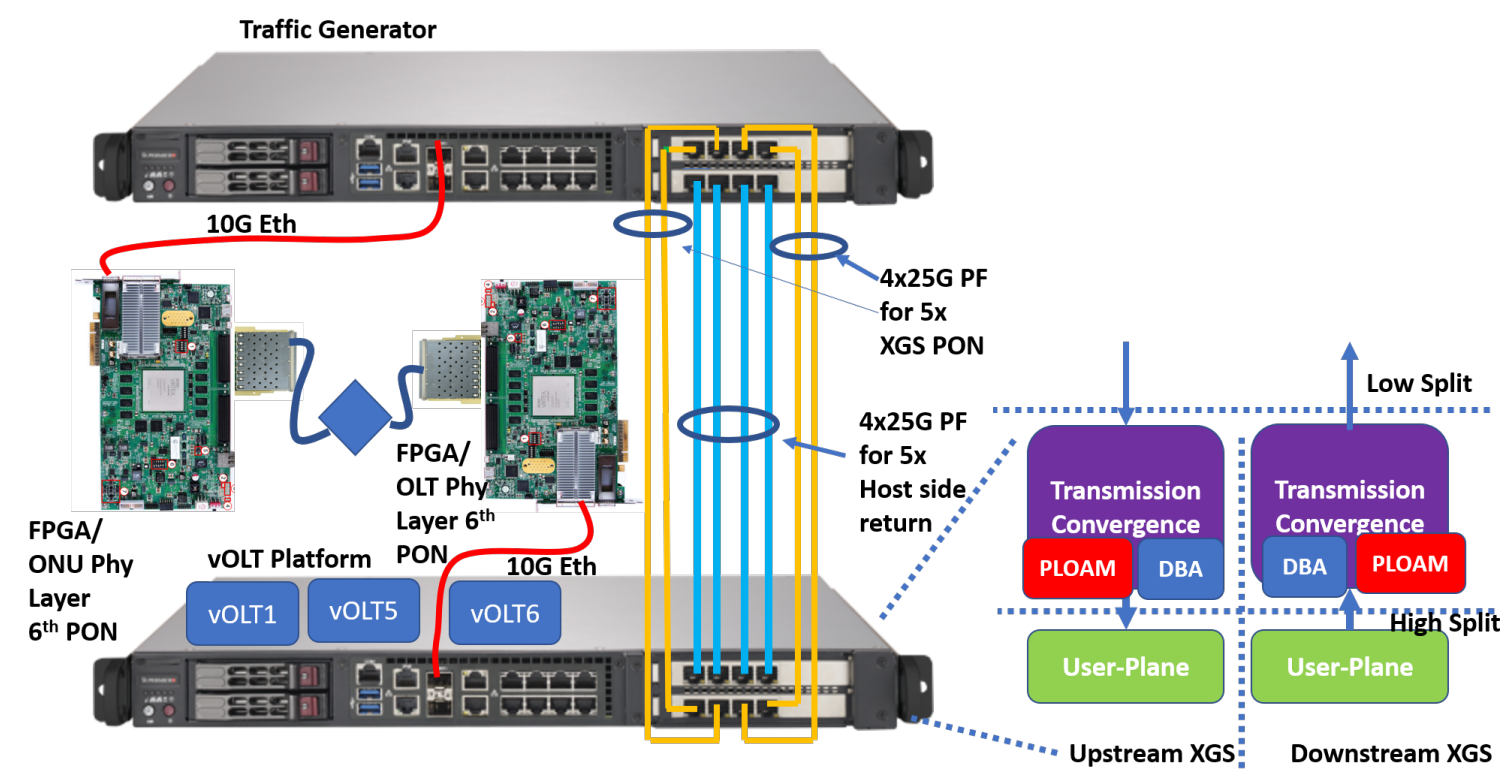

Fig. 1. Demo configuration of True Real Time Multi-Tenant PON based on vOLT and vDBA

The demonstration set-up in Fig. 1, is composed of the common virtual OLT (vOLT) server, a physical PON and a traffic generation Node. The demonstration runs on the the Intel ${ }^{\circledR}$ vOLT reference software, which consists of a DPDK-based vOLT reference application and DPDK-Pktgen-based traffic generator. The vOLT application provides OLT data-plane and basic PON management processing based on Broadband Forum (BBF) and ITU-T XGS-PON standards. An instance of the vOLT application runs for each PON port on the system. Version 19.07

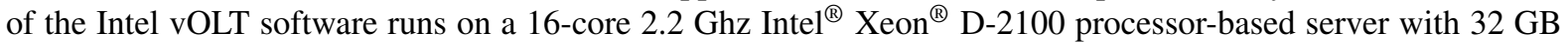
DDR4 DRAM and Silicom Quad Port PCIe16 Gen3 Server adapters. 
The virtual OLT software can be configured to run in two modes:

- High Split Mode: where the XG-PON transmission convergence layer (XGTC) and XG-PON encapsulation method (XGEM) framing is done in hardware and the User-Plane in software;

- Low Split Mode: where XGTC, XGEM framing and User-Plane all run in software.

For this demonstration, the PONs run in low split mode with the physical layer of 5 of the PONs emulated by encapsulating the XGTC/FS frame in ethernet. The physical layer of the 6th PON is instead executed on two VCU108 FGPA boards connected through a length of optical fibre, providing a PON physical layer where the OLT can operate in burst-mode reception. Fig. 2 shows the allocation of the CPU Cores to the vOLT and vDBA

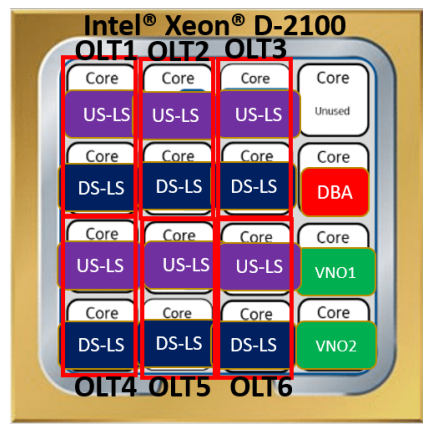

Fig. 2. Allocation of cores to vOLT and vDBA functions. US=upstream; DS=downstream

functions. 2 cores are allocated to the Upstream and Downstream paths of each vOLT. A single core is allocated to the Virtual Dynamic Bandwdith Assignment (vDBA) Merging Engine, which merges all virtual bandwidth maps from the different VNOs and generates a single physical bandwidth Map allocation. A single core is allocated to the execution of each Virtual Network Operator (VNO). The traffic generator runs a DPDK optimised Pktgen on an 18-core $2.3 \mathrm{Ghz}$ Intel ${ }^{\circledR} \mathrm{Xeon}^{\circledR}$ Scaleable Processor-based server with 96 GB DDR4 RAM and 4xDual 25G NICs.

The scenarios which we demonstrate are as follows. For all vOLTs, 64 Optical Network Terminals (ONTs) are configured with 4 downstream Traffic Classes related to Management, Voice, Video and High Speed Internet. For the 5 vOLT, where the physical layer of the PONs is emulated by encapsulating the XGTC/FS frame in ethernet, there are 8 Traffic Containers (T-CONTs) defined with an even amount of bandwidth allocated by the vDBA. For the 6th vOLT with the physical transmission layer, we distribute the 64 ONTs and the corresponding upstream bandwidth across two VNOs in the ratio of 48:16. Our virtual DBA solution allows each VNO to run its own independent DBA algorithm for its own ONTs. The Merging Engine aggregates all individual virtual Bandwidth Maps (BWmaps) generates by the VNOs into a single BWmap that is physically distributed to all ONTs.
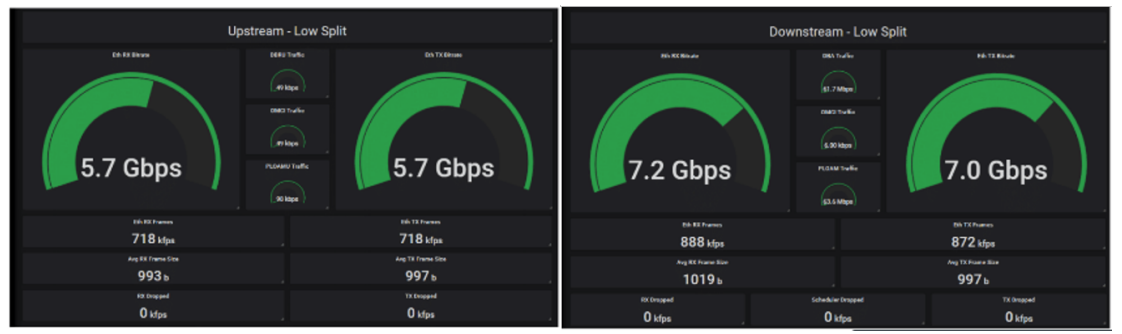

Fig. 3. Upstream and Downstream throughput (Gbps) for fully softwarised OLT

The graphs for downstream and upstream throughput for each of the vOLT are shown in Fig. 3. For each direction, the traffic transmitted and received is shown. $5.7 \mathrm{Gbps}$ is sent and received by the traffic generator in the upstream direction. In the downstream direction, $7.0 \mathrm{Gbps}$ is sent and $7.2 \mathrm{Gbps}$ is received, the difference of 200Mbps being accounted by PLOAM and DBA data. It should be noticed that such rates were achieved by running the Low Split Mode, which implies full softwarisation of the data plane, including upstream and downstream scheduling algorithms. Through further optimisation of cache management by Intel, we believe that throughput for both upstream and downstream may be improved to $8.55 \mathrm{Gbps}$. 


\section{Novelty}

Our demonstration is unique, in that we deliver a fully virtualised XGS-PON compliant [2,3] OLT and DBA that runs on General Purpose Processor. In contrast, in the OpenOLT project [1] which disaggregates the OLT into MAC (data plane) and the control layers, the data plane is typically based on the Broadcom BCM68620 chipset which provides both XG-PON and NGPON2 mac layers in hardware. An example of the OpenOLT disaggregated OLT, is the Edgecore ASXvOLT16. The implementation of the MAC layer in hardware means that functions such as the DBA function are fixed and thus cannot be offloaded to a virtualised environment, thereby restricting upgradeability and innovation in slicing.

The principle of virtualising the DBA function, where the DBA is moved from dedicated hardware to software, was first introduced in [4] and demonstrated in [5]. The importance of vDBA is that it gives VNOs control, at the individual physical frame level, of the upstream capacity allocation in PONs. This is essential for enforcing strict Quality of Service (QoS) parameters, including low-latency, in a shared network environment. Control over capacity assignment algorithms guarantees the quality of service level required by future $5 \mathrm{G}$ services and Fixed Access Network Sharing [6]. This contrasts with traditional access network sharing which has been regulatory driven and implemented at the higher layers of the telecommunication stack.

The performance of the virtualised OLT and DBA is greatly improved through the use of the DPDK dataplane acceleration toolkit. DPDK offers user space drivers and libraries, providing high performance direct access from physical devices to user space processes. DPDK also uses packet processing optimization techniques such as zero copy, batch processing of packets, buffer allocations, interrupt-less I/O and processor affinity to reduce the overhead of running the OLT and DBA processes on a shared system.

The low-split mode of the vOLT software, where all OLT functions run in software and are thus fully disaggregated is also key for the development of novel access architectures based on Multi-Access Edge Computing (MEC), giving the flexibility to locate such functions also outside the main central office. For instance, the lower layer of the vOLT could be located as a remote PHY module, as part of an extended reach PON, with the User Plane centrally located, to better suit the economics of rural Fibre to the Home (FTTH) deployment.

\section{OFC Relevance}

This presentation is highly relevant to the OFC SDN \& NFV event as it demonstrates a fully virtualised XGS-PON compliant [2,3] OLT and DBA as part of a fully sliceable PON. The demonstration is carried out between the Intel Corporation Ireland, which developed the virtual OLT and the TCD Optical Network Architecture groups, which developed the DBA virtualisation and merging concepts and algorithms. This topic is timely and highly relevant to industry, which is currently standardising the framework for Fixed Access Network Sharing (FANS) [6], cloud Central Office (cloud-CO) [7], as well as use of interfaces for OLT virtualisation [8]. The demonstration we propose is relevant to vendors, operators and service providers, that are interested in implementing the NFVdriven central office virtualisation, extending the R-CORD implementation to a solution that fully virtualises the OLT data plane. The multi-service and multi-tenant aspects delivered by our solutions are key to supporting the highly heterogeneous services and applications envisaged by the $5 \mathrm{G}$ and beyond vision.

\section{Acknowledgments}

Financial support from Science Foundation Ireland (SFI) grants 14/IA/2527 (O'SHARE) and 13/RC/2077 (CONNECT) is gratefully acknowledged.

\section{References}

1. Al-Shabibi et. al, 'Virtual OLT (vOLT). CORD Design Notes' http://opencord. org/wpcontent/uploads/2016/03/Virtual-OLT.pdf, 2016.

2. ITU-T G.9807.1, "10 Gigabit-capable passive optical networks", 2015

3. BBF TR-156, "Using GPON Access in the Context of TR-101", 2012. https://www. broadband-forum.org/download/TR-156.pdf

4. Elrasad et. al, "Virtual Dynamic Bandwidth Allocation Enabling True PON Multi-Tenancy," OFC, 2017.

5. Slyne et. al, Demonstration of real time VNF implementation of OLT with virtual DBA for sliceable multitenant PONs. OFC 2018.

6. BBF TR-370, "Fixed Access Network Sharing - Architecture and Nodal Requirements", 2017. https: //www.broadband-forum.org/download/TR-370.pdf

7. BBF TR-384, "Cloud Central Office Reference Architectural Framework", 2018, https://www. broadband-forum.org/download/TR-384.pdf.

8. BBF TR-402, "Functional Model for PON Abstraction Interface", 2018. https://www. broadband-forum.org/download/TR-402.pdf. 